\section{NachwuchsFörderPreis}

Dr. Martin Diers, Mannheim, Dr. Chrsitoph Ziskoven, Düsseldorf, und Dr. Kirsten Kempf, Düsseldorf wurden für ihre herausragenden wissenschaftlichen Arbeiten auf dem Gebiet der Schmerztherapie mit dem NachwuchsFörderPreis Schmerz ausgezeichnet. Der Preis wird jährlich für besondere wissenschaftliche Leistungen im Bereich der chronischen und postoperativen Schmerztherapie vergeben. Die drei besten Arbeiten werden mit 5.000 Euro, 2.000 Euro und 1.000 Euro prämiert. „Es ist uns als Initiatoren des Preises ein Anliegen, die innovativen richtungweisenden Forschungsprojekte junger aufstrebender Talente, die am Anfang ihrer Karriere stehen, zu würdigen und zu fördern (...)", unterstrichen PD Dr. Thorsten Gieseke und Dr. Antonie Wimmer stellvertretend für das stiftende Unternehmen Janssen bei der Preisverleihung im Mai in Berlin.

Nach Information von Janssen-Cilag

Periphere Neuropathie: Hochdosierte Capsaicin-Pflaster

Neuropathische Schmerzen sind besonders schwierig zu behandeln. Eine neue Therapieoption für Patienten mit peripheren neuropathischen Schmerzen ist ein Pflaster mit $8 \%$ Capsaicin (Qutenza ${ }^{\mathrm{TM}}$ ), das bei einmaliger Anwendung bis zu zwölf Wochen wirkt. In den klinischen Studien sprachen bis zu $45 \%$ der Behandelten auf die topische Therapie an. Capsaicin stimuliert den Vanilloidrezeptor 1 (TRPV1 ), der von afferenten $C$ - und $A \delta$ - Nervenfasern exprimiert wird und bei neuropathischen Schmerzen überaktiv ist. Durch Capsaicin 8\% werden die epidermalen Nervenfasern vorübergehend defunktionalisiert, erklärte PD Dr. Michael Überall, Nürnberg. Die Folge ist eine deutliche, bis zu zwölf Wochen anhaltende Schmerzlinderung, wie klinische Studien bei insgesamt mehr als 2.300 Patienten mit Mononeuropathien belegen. Zugelassen ist das hoch dosierte Capsaicinpflaster zur Behandlung von Erwachsenen mit postherpetischer und HIV-Neuropathie, die nicht an Diabetes erkrankt sind. Die topische Therapie ist gut mit oralen Medikamenten zu kombinieren und verursacht keine systemischen Nebenwirkungen.

Symposium "Neuropathischer Schmerz häufig unterschätzt?!", im Rahmen des Deutschen Schmerz- und Palliativtages 2011, Frankfurt am Main, 24. März 2011, Veranstalter: Astellas; Bericht: Roland Fath, Frankfurt/ Main

\title{
Neuartige Galenik von Fentanyl ermöglicht Einsatz als schnelle Bedarfsmedikation
}

— Das Fentanyl-Präparat (Effentora ${ }^{\circledR}$ Buccaltabletten) von Cephalon lindert Durchbruchschmerzen bei Tumorschmerzpatienten schnell und anhaltend - und ist damit eine sehr effektive Bedarfsmedikation bei Tumorschmerzen. Die lokalen pH-Wert-Veränderungen an der Wangenschleimhaut durch die bei den Buccaltabletten genutzte OraVescent ${ }^{\oplus}$-Technologie sind der Schlüssel für die optimierte Resorption von Fentanyl: OraVescent ${ }^{\oplus}$-Technologie verbessert die buccale Fentanyl-Resorption.

- Bei Kontakt mit Speichel kommt es zu einer leichten Brausereaktion mit Bildung von Kohlensäure.

- Dadurch sinkt am Applikationsort der pH-Wert, was die Auflösung der Buccaltablette und die Fentanyl-Freisetzung fördert.

- Danach steigt der pH-Wert infolge der Dissoziation von Kohlensäure in Kohlendioxid $\left(\mathrm{CO}_{2}\right)$ und Wasser wieder an, $\mathrm{CO}_{2}$ entweicht.

- Bedingt durch die pH-Wert-Erhöhung (in den basischen Bereich) geht Fentanyl in die nicht ionisierende, lipophilere Form über, die leicht an die lipophilen Moleküle der Mundschleimhaut bindet.

- Auf diese Weise wird die Permeation durch die Mundmukosa erleichtert und somit die Fentanyl-Aufnahme in den Blutkreislauf beschleunigt.

Die lipophile Substanz kann die Blut-HirnSchranke schnell passieren und seine analgetische Wirkung im ZNS entfalten. Die Schmerzlinderung wird innerhalb von zehn Minuten spürbar. Etwa die Hälfte der buccal applizierten Dosis wird transmukosal aufgenommen. Das übrige Fentanyl wird durch Schlucken über den Gastrointestinaltrakt aufgenommen und unterliegt dem Firstpass-Metabolismus in der Leber, sodass davon etwa ein Drittel systemisch wirksam wird. Somit beträgt die absolute Bioverfügbarkeit der applizierten Fentanyldosis insgesamt etwa $65 \%$.

Zugelassen sind die Fentanyl-Buccaltabletten zur Behandlung von Durchbruchschmerzen bei erwachsenen Krebspatienten, die wegen ihrer chronischen Tumorschmerzen bereits eine Opioid-Basistherapie erhalten. Das Medikament wird von Cephalon und AWD. pharma als Co-Marketing-Partner in fünf

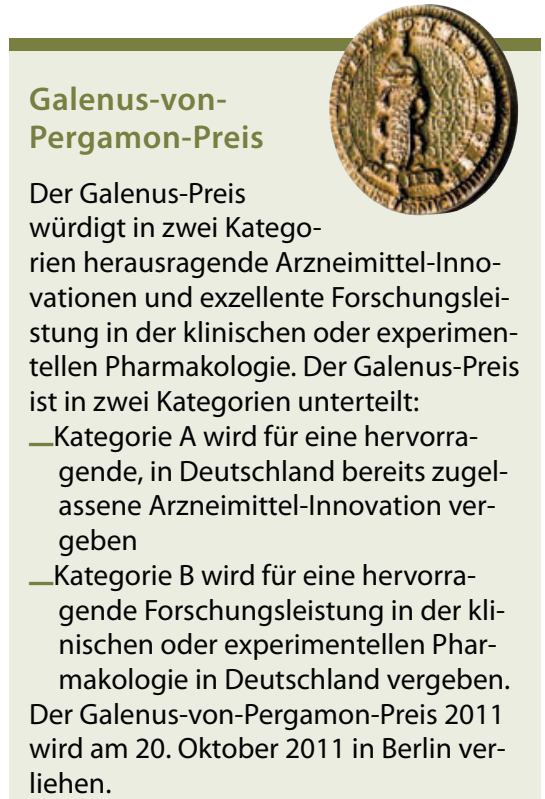

Cephalon bewirbt sich mit Effentora ${ }^{\circledR}$ in diesem Jahr für den Preis.

Wirkstärken - mit $100 \mu \mathrm{g}, 200 \mu \mathrm{g}, 400 \mu \mathrm{g}$, $600 \mu \mathrm{g}$ und $800 \mu \mathrm{g}$ Fentanyl - angeboten. Die schnelle schmerzlindernde Wirkung des Medikaments wurde in mehreren Studien bei Krebskranken mit Durchbruchschmerzen belegt. In einer placebokontrollierten, doppelblinden Studie bei 87 Patienten war die Schmerzlinderung bereits zehn Minuten nach Anwendung der Fentanyl-Buccaltabletten in individuell erforderlicher Wirkstärke, die zuvor in einer offenen Studienphase austitriert worden war, signifikant besser als mit Placebo. Primärer Studienendpunkt war die Summe der Schmerzstärke (PI)-Differenzen zwischen 5 und 60 Minuten. Mit Fentanyl war die PIDifferenz schon nach 10 Minuten signifikant größer, sie nahm innerhalb von 90 Minuten weiter zu und blieb bis zum letzten Messzeitpunkt (nach 120 Minuten, sekundärer Endpunkt) konstant. Eine zusätzliche Bedarfsmedikation war nur bei $11 \%$ der Durchbruchschmerz-Episoden, bei denen mit Fentanyl behandelt wurde, erforderlich, aber bei 30\% der Episoden mit Placebo-Therapie.

Die Therapie mit Fentanyl-Buccaltabletten war mit allen Wirkstärken sicher; auftretende Nebenwirkungen (UAW) waren, wie erwartet, opioidtypisch (etwa Benommenheit, Übelkeit, Erbrechen).
Ulrike Maronde 